\title{
Wake structure and thrust generation of a flapping foil in two-dimensional flow
}

\author{
Andersen, Anders Peter; Bohr, Tomas; Schnipper, Teis; Walther, Jens Honore
}

Published in:

Journal of Fluid Mechanics

Link to article, DOI:

10.1017/jfm.2016.808

Publication date:

2017

Document Version

Peer reviewed version

Link back to DTU Orbit

Citation $(A P A)$ :

Andersen, A. P., Bohr, T., Schnipper, T., \& Walther, J. H. (2017). Wake structure and thrust generation of a flapping foil in two-dimensional flow. Journal of Fluid Mechanics, 812, [R4]. https://doi.org/10.1017/jfm.2016.808

\section{General rights}

Copyright and moral rights for the publications made accessible in the public portal are retained by the authors and/or other copyright owners and it is a condition of accessing publications that users recognise and abide by the legal requirements associated with these rights.

- Users may download and print one copy of any publication from the public portal for the purpose of private study or research.

- You may not further distribute the material or use it for any profit-making activity or commercial gain

- You may freely distribute the URL identifying the publication in the public portal

If you believe that this document breaches copyright please contact us providing details, and we will remove access to the work immediately and investigate your claim. 


\title{
Wake structure and thrust generation of a flapping foil in two-dimensional flow
}

\author{
By A. A NDERSEN ${ }^{1} \dagger$, T. B O HR $\mathbf{R}^{1}$, T. SCHNIPPER $\mathbf{R}^{1,2} \ddagger$ \\ AND J. H. W A L T H E R $\mathbf{R}^{2,3}$ \\ ${ }^{1}$ Department of Physics and Center for Fluid Dynamics, \\ Technical University of Denmark, DK-2800 Kgs. Lyngby, Denmark \\ ${ }^{2}$ Department of Mechanical Engineering and Center for Fluid Dynamics, \\ Technical University of Denmark, DK-2800 Kgs. Lyngby, Denmark \\ ${ }^{3}$ Swiss Federal Institute of Technology Zürich, Chair of Computational Science, \\ ETH Zentrum, Clausiusstrasse 33, CH-8092 Zürich, Switzerland
}

(Received 11 November 2016)

We present a combined numerical (particle vortex method) and experimental (soap film tunnel) study of a symmetric foil undergoing prescribed oscillations in a two-dimensional free stream. We explore pure pitching and pure heaving and contrast these two generic types of kinematics. We compare measurements and simulations when the foil is forced with pitching oscillations, and we find a close correspondence between flow visualisations using thickness variations in the soap film and the numerically determined vortex structures. Numerically we determine wake maps spanned by oscillation frequency and amplitude, and we find qualitatively similar maps for pitching and heaving. We determine the drag-thrust transition for both pitching and heaving numerically, and we discuss it in relation to changes in wake structure. For heaving with low oscillation frequency and high amplitude we find that the drag-thrust transition occurs in a parameter region with wakes in which two vortex pairs are formed per oscillation period in contrast to the common transition scenario in regions with inverted von Kármán wakes.

\section{Introduction}

Oscillating foils can lead to the formation of a variety of spectacular vortex patterns (Koochesfahani 1986, 1989; Schnipper et al. 2010), and such flows and the forces that generate them are at the same time of great significance in fluid-structure interactions, e.g., for understanding the function of swimming appendages in biological locomotion (Lighthill 1969; Triantafyllou et al. 2000). The kinematics and dynamics of swimming appendages in nature is typically complex, involving a combination of pitching and heaving (Sfakiotakis et al. 1999), effects of foil flexibility (Marais et al. 2012; Jaworski \& Gordnier 2015), and three-dimensional flows (Buchholz \& Smits 2008).

In the present paper we consider a rigid foil with prescribed flapping motion in a twodimensional free stream to explore basic aspects of wake formation and the associated fluid forces. Experimentally and numerically we consider two generic types of kinematics, pure pitching and pure heaving, respectively. We used a gravity-driven, vertically flowing soap film for our experimental study (Rutgers et al. 2001), and the experimental set-up and main observations were first presented by Schnipper et al. (2009). Our simulations of

$\dagger$ Email address for correspondence: aanders@fysik.dtu.dk

$\ddagger$ Present address: Svend Ole Hansen ApS, Sct. Jørgens Allé 5 C, DK-1615 Copenhagen 
$(a)$

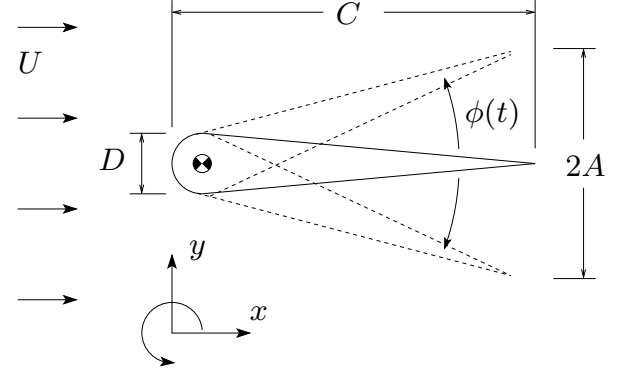

(b)

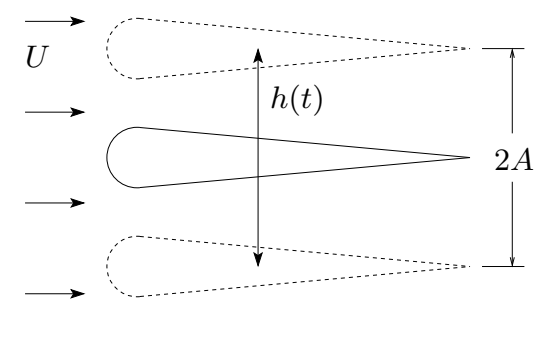

FiguRE 1. Geometry and kinematics of the foil. (a) pure pitching and (b) pure heaving.

two-dimensional, incompressible Newtonian flow were carried out using a particle vortex method (Walther \& Larsen 1997), and the numerical findings have not been presented previously. The round leading edge and the wedge-shaped trailing edge of the symmetric foil give rise to two distinct sources of vorticity, i.e., the boundary layers along the two sides of the foil and the trailing edge, leading to formation of simple or complex vortex wakes depending on the oscillation frequency and amplitude.

Soap film tunnels have been used as simple and powerful flow visualisation tools for a variety of vortex flows (Couder \& Basdevant 1986; Gharib \& Derango 1989; Rivera et al. 1998; Zhang et al. 2000), but only few studies have explored to what extent soap film flows provide a quantitative analogy with two-dimensional, incompressible Newtonian flows (Couder et al. 1989; Chomaz \& Cathalau 1990). We therefore explore the relation between the vortex structures visualized experimentally using thickness variations in the quasi two-dimensional soap film flows and the vortex structures in the corresponding two-dimensional, incompressible Newtonian flows obtained numerically.

Further we contrast the wake formation in the two kinematic modes using the numerical simulations, and we consider the net fluid forces on the flapping foil with particular focus on the drag-thrust transition and its relation to changes in wake structure. This problem has been investigated previously for pure pitching motion in experiments in which the drag-thrust transition was inferred from flow field measurements (Godoy-Diana et al. 2008; Bohl \& Koochesfahani 2009), in direct measurements using force transducers (Mackowski \& Williamson 2015), and in numerical simulations (Das et al. 2016). We add to these studies by comparing the drag-thrust transition in the two different kinematic modes under similar flow conditions, and we find an atypical transition scenario for the heaving foil at low oscillation frequency and high amplitude.

\section{Foil kinematics and dimensionless parameters}

The foil is rigid and has a semi-circular leading edge with diameter $D$, chord length $C$, aspect ratio $D / C=1 / 6$ and straight sides that form a wedge-shaped trailing edge (Schnipper et al. 2009). The foil is placed in a uniform inflow with speed $U$ in the $x$ direction and it is oscillated with frequency $f$ and amplitude $A$. In the pure pitching mode (figure 1a), the foil pitches around the centre of the semi-circular leading edge such that the foil angle $\phi$ with respect to the free-stream direction varies with the simple harmonic motion

$$
\phi(t)=\phi_{0} \sin 2 \pi f t
$$

where the amplitudes $\phi_{0}$ and $A$ are connected by the relation $\sin \phi_{0}=12 A / 11 C$. In 
the pure heaving mode (figure 1b), the foil oscillates transversely to the free-stream such that the $y$-coordinate of the centre line of the foil $h$ follows the equation

$$
h(t)=A \sin 2 \pi f t
$$

The oscillating foil in the two-dimensional, incompressible Newtonian flow is characterised by three dimensionless parameters, i.e., the width-based Strouhal number

the dimensionless amplitude

$$
\mathrm{St}_{D}=\frac{D f}{U}
$$

$$
A_{D}=\frac{2 A}{D}
$$

and the Reynolds number based on the chord length $\operatorname{Re}=C U / \nu$, where $\nu$ is the kinematic viscosity of the fluid. The width-based Strouhal number $\mathrm{St}_{D}$ describes the externally imposed frequency and not the natural vortex shedding frequency, and it is sometimes referred to as the Keulegan-Carpenter number. The product of $\mathrm{St}_{D}$ and $A_{D}$ is the so-called amplitude-based Strouhal number

$$
\mathrm{St}_{A}=\frac{2 A f}{U}
$$

The amplitude-based Strouhal number can be understood as the ratio between the speed of the foil tip and the speed of the free-stream, and it plays a central role in describing the drag-thrust transition (Triantafyllou et al. 1991).

\section{Particle vortex method}

The numerical simulations were performed using a two-dimensional discrete particle vortex method. The approach is described in detail elsewhere (Walther \& Larsen 1997; Rasmussen et al. 2010), and it has been validated for the flow past a flat plate (Walther \& Larsen 1997; Hejlesen et al. 2015) and for the flow past suspension bridge sections (Larsen \& Walther 1997). The method is based on a Lagrangian formulation of the two-dimensional vorticity equation

$$
\frac{\partial \omega}{\partial t}+(\vec{v} \cdot \nabla) \omega=\nu \nabla^{2} \omega
$$

where $\vec{v}$ denotes the velocity and $\vec{\omega}=\nabla \times \vec{v}$ the vorticity. At each time-step the instantaneous fluid velocity is obtained from the Biot-Savart relation

$$
\begin{aligned}
\vec{v}(x, y) & =\vec{U}+\frac{1}{2 \pi} \iint_{S_{\mathrm{fluid}}} \frac{\vec{\omega}\left(x^{\prime}, y^{\prime}\right) \times\left(\vec{x}-\vec{x}^{\prime}\right)}{\left|\vec{x}-\vec{x}^{\prime}\right|^{2}} \mathrm{~d} x^{\prime} \mathrm{d} y^{\prime} \\
& -\frac{1}{2 \pi} \oint_{C_{\text {foil }}} \frac{\left[\vec{v}\left(x^{\prime}, y^{\prime}\right) \cdot \vec{n}\left(x^{\prime}, y^{\prime}\right)\right]\left(\vec{x}-\vec{x}^{\prime}\right)-\left[\vec{v}\left(x^{\prime}, y^{\prime}\right) \times \vec{n}\left(x^{\prime}, y^{\prime}\right)\right] \times\left(\vec{x}-\vec{x}^{\prime}\right)}{\left|\vec{x}-\vec{x}^{\prime}\right|^{2}} \mathrm{~d} l^{\prime}
\end{aligned}
$$

where $\vec{n}$ denotes the outward unit normal at the foil boundary, $C_{\text {foil }}$ the foil boundary and $S_{\text {fluid }}$ the fluid domain. The boundary integral accounts for the vorticity within the foil due to the solid body rotation. The foil boundary is discretised using boundary elements with vortex sheets of linearly varying strength. In the present study we discretise the foil boundary using 400 elements and conduct the simulations for 10 periods of 

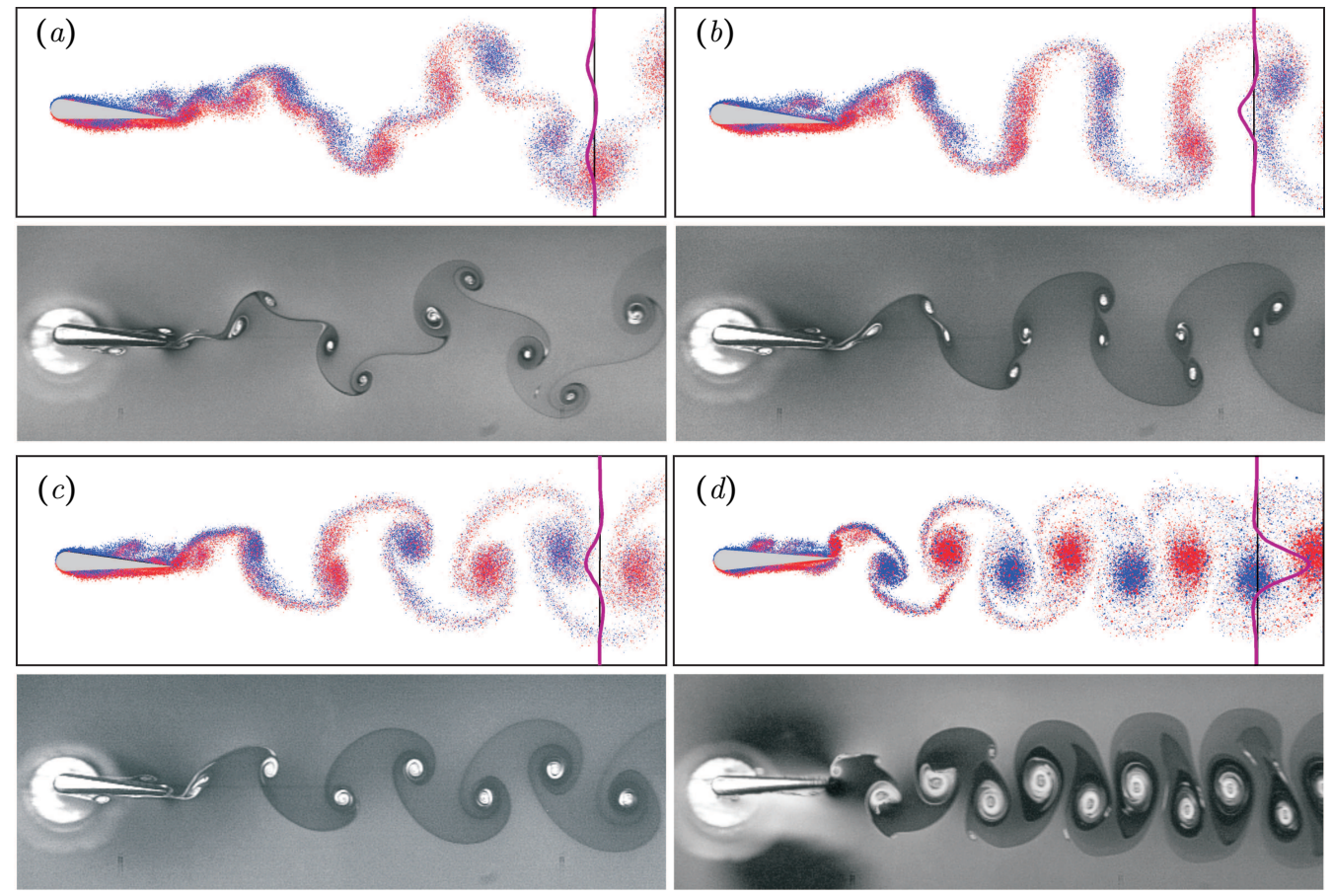

FIGURE 2. Wakes of the pitching foil in simulation (colour) and experiment (grey-scale). Red (blue) corresponds to positive (negative) vorticity, and the magenta lines show average velocity profiles four chord lengths downstream of the trailing edge with the black lines showing the inflow velocity as reference. (a) $2 P$ wake $\left(\mathrm{St}_{D}=0.08, A_{D}=1.14\right.$, and $\left.\left\langle C_{\mathrm{T}}\right\rangle=-0.12\right)$, see also supplementary video $1,(b) 2 P$ wake that evolves to a von Kármán wake $\left(\mathrm{St}_{D}=0.11, A_{D}=1.14\right.$, and $\left.\left\langle C_{\mathrm{T}}\right\rangle=-0.12\right),(c)$ von Kármán wake $\left(\mathrm{St}_{D}=0.12, A_{D}=1.14\right.$, and $\left.\left\langle C_{\mathrm{T}}\right\rangle=-0.12\right)$ and $(d)$ inverted von Kármán wake $\left(\mathrm{St}_{D}=0.18, A_{D}=1.65\right.$, and $\left.\left\langle C_{\mathrm{T}}\right\rangle=0.00\right)$.

the flapping motion. The no-slip boundary condition is imposed by solving a boundary integral equation obtained from equation (3.2) for the unknown vortex sheet strengths while satisfying Kelvin's circulation theorem. The vortex sheets are subsequently diffused into the flow to form the no-slip boundary layer. The advection is described by the inviscid motion of the discrete vortex particles and viscous diffusion is modelled using the method of random walks (Chorin 1973). In the simulations we have $\mathrm{Re}=2640$. To reduce computational costs, the velocity equation (3.2) is computed using the adaptive fast multipole method (Carrier et al. 1988), and vortex particles are amalgamated five chordlengths downstream of the trailing edge. Control simulations without amalgamation show that amalgamation has no effect on the fluid forces acting on the foil.

The instantaneous net fluid force on the foil is computed from the equation

$$
\vec{F}(t)=\rho \frac{\mathrm{d}}{\mathrm{d} t}\left(\iint_{S_{\text {foil }}} \vec{v} \mathrm{~d} x \mathrm{~d} y-\iint_{S_{\text {fluid }}} \vec{x} \times \vec{\omega} \mathrm{d} x \mathrm{~d} y\right),
$$

where $\rho$ is the density of the fluid and $S_{\text {foil }}$ the domain occupied by the foil (Wu 1981; Walther \& Larsen 1997). We shall for convenience decompose the net fluid force on the foil in thrust and lift components $F_{\mathrm{T}}=-F_{x}$ and $F_{\mathrm{L}}=F_{y}$, and use the standard definitions of the instantaneous thrust and lift coefficients 


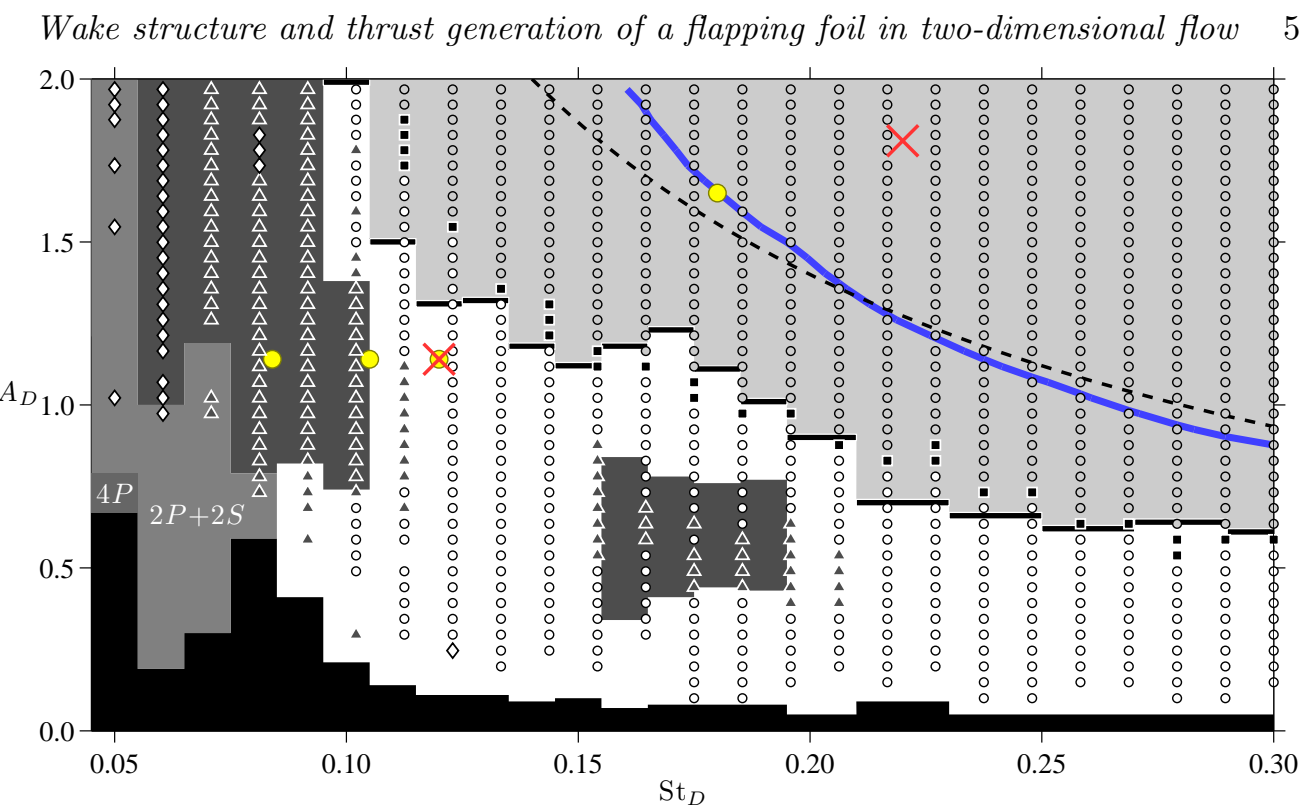

FIGURE 3. Wake map for the pitching foil. Experiment (background shades) and simulation (symbols). (०, white) von Kármán wakes, (•, light grey) inverted von Kármán wakes, (๓, black bars) aligned $2 S$ wakes with vortices on the symmetry line, ( $\boldsymbol{\Delta}$, dark grey) $2 P$ wakes and $(\diamond)$ periodic vortex wakes with blurry vorticity regions. The yellow discs indicate the cases in figure 2 and the red crosses the examples shown in figure 4. The solid blue curve shows the drag-thrust transition, and the dashed black curve shows the contour line with $\mathrm{St}_{A}=0.28$.

$$
C_{\mathrm{T}}=\frac{F_{\mathrm{T}}}{(1 / 2) \rho C U^{2}}, \quad C_{\mathrm{L}}=\frac{F_{\mathrm{L}}}{(1 / 2) \rho C U^{2}} .
$$

From equation (3.3) we obtain the equation for the thrust component

$$
F_{\mathrm{T}}=-\rho \frac{\mathrm{d}}{\mathrm{d} t}\left(\iint_{S_{\text {foil }}} v_{x} \mathrm{~d} x \mathrm{~d} y-\iint_{S_{\text {fluid }}} y \omega \mathrm{d} x \mathrm{~d} y\right) .
$$

The equation highlights the close connection between the transition from drag to thrust and the transition in the arrangement of the coherent vortices in the wake, e.g., from von Kármán wake with $y$ and $\omega$ predominantly of opposite signs to inverted von Kármán wake with $y$ and $\omega$ predominantly of the same sign (von Kármán \& Burgers 1935).

\section{Pitching foil in experiment and simulation}

We first consider the pitching foil in experiment and simulation. We shall label the wakes using the symbols introduced by Williamson \& Roshko (1988), where $m S+n P$ signifies $m$ single vortices and $n$ vortex pairs shed per oscillation period. Figure 2 shows a $2 P$ wake $(a)$, a $2 P$ wake that evolves downstream to a von Kármán wake $(b)$, a von Kármán wake $(c)$, and an inverted von Kármán wake $(d)$. In the simulation images, each dot corresponds to a vortex particle with positive (red) or negative (blue) circulation. Regions with high dot density that are dominated by red (blue) dots correspond to vortices of positive (negative) net circulation. The wake types are identified by visual inspection in the approximate interval 2-4 chord lengths downstream of the trailing 


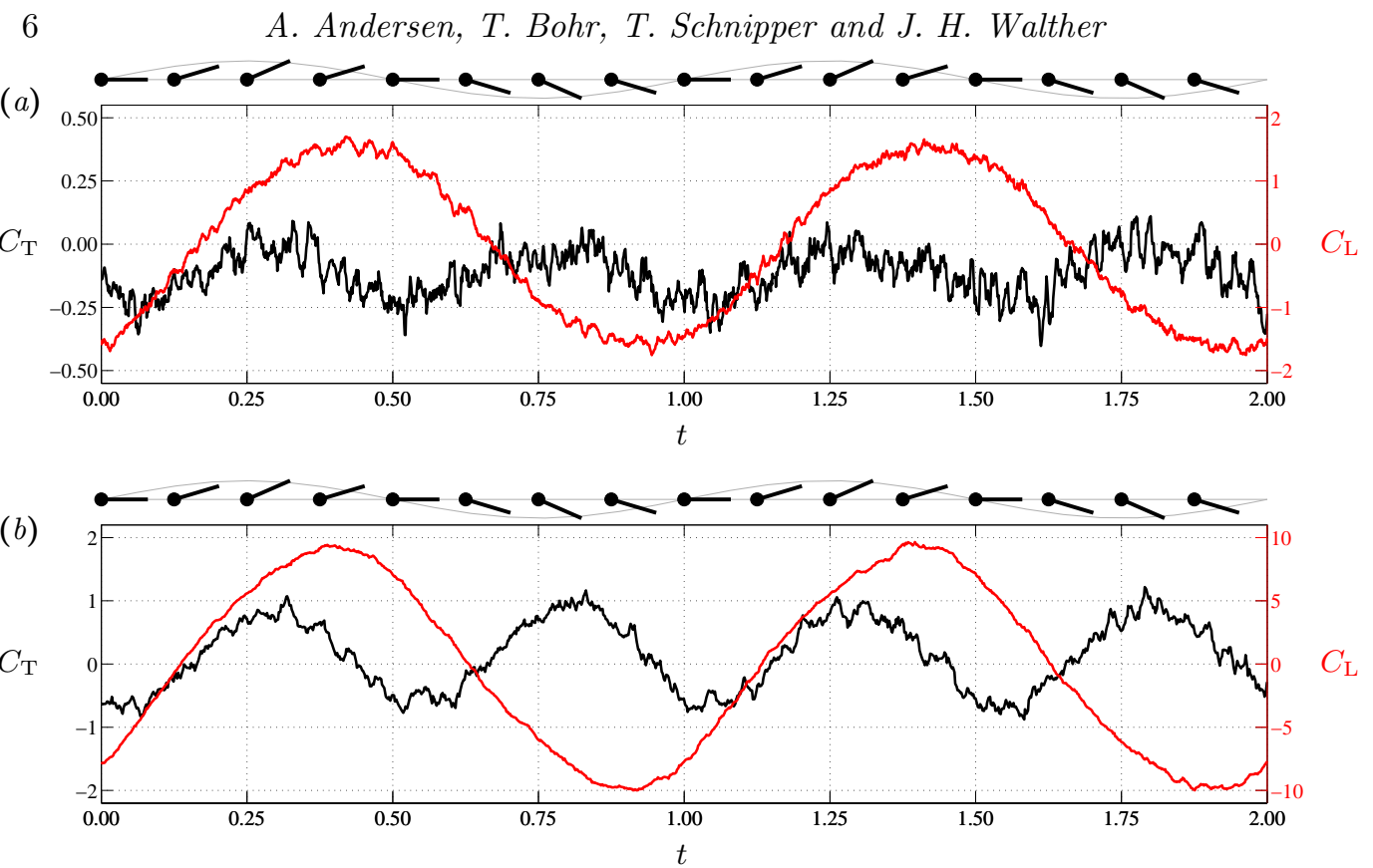

Figure 4. Instantaneous thrust (black) and lift (red) coefficients for the pitching foil in the simulation. ( $a$ ) von Kármán wake (cf. figure $2 c$ ) with average drag $\left(\mathrm{St}_{D}=0.12\right.$ and $A_{D}=1.14$ ) and $(b)$ inverted von Kármán wake with average thrust $\left(\mathrm{St}_{D}=0.22\right.$ and $\left.A_{D}=1.81\right)$.

edge, and the soap film experiment additionally allows us to use a long 20 chord length interval to follow the downstream wake evolution.

In all four cases we observe that the vortex particles in the simulation and the thickness variations in the soap film form similar structures, i.e., concentrated vortices connected by narrow, curved bands of vorticity. Agreement is also observed in the flow close to the leading and trailing edges, where four patches of vorticity form in every oscillation period. For example, both methods show formation of two vortex patches, one on each side of the leading edge, per flapping period. After formation, the patches roll downstream along the foil (Schnipper et al. 2009). In figure 2(b) we plot an intermediate wake type in which a $2 P$ wake evolves downstream to a von Kármán wake by pairwise merging of vortices. We note that a slight decrease of $\mathrm{St}_{D}$ yields a stable $2 P$ wake whereas a slight increase yields a von Kármán wake. Differences between simulation and experiment are also observed: The vortices in the inverted von Kármán wake in figure $2(d)$ are located $18 \%$ closer to each other in the experiment than in the simulation. We presume that this is due to the relatively intense flapping of the foil which gives rise to compressibility effects in the soap film flow (Chomaz \& Cathalau 1990).

The four wakes in figure 2 are representative of the most significant wake types in the wake map (phase diagram) in figure 3 . The symbols indicating periodic wake types in the simulation are plotted on top of the corresponding wake map (shaded areas) measured in the soap film (Schnipper et al. 2009). Wakes with no clear periodicity were found in the simulations where dots are absent in the phase diagram and in the soap film where the background shade is black. Generally, there is a striking overall agreement in the variety of vortex wakes. Both methods find a region $\left(\mathrm{St}_{D}>0.1\right)$ that is dominated by $2 S$ wake types as observed by Godoy-Diana et al. (2008), and a region $\left(\mathrm{St}_{D}<0.1\right)$ that is dominated by complex wakes. We observe that the boundaries between von Kármán wake and inverted von Kármán wake correspond well to each other, and only at low 

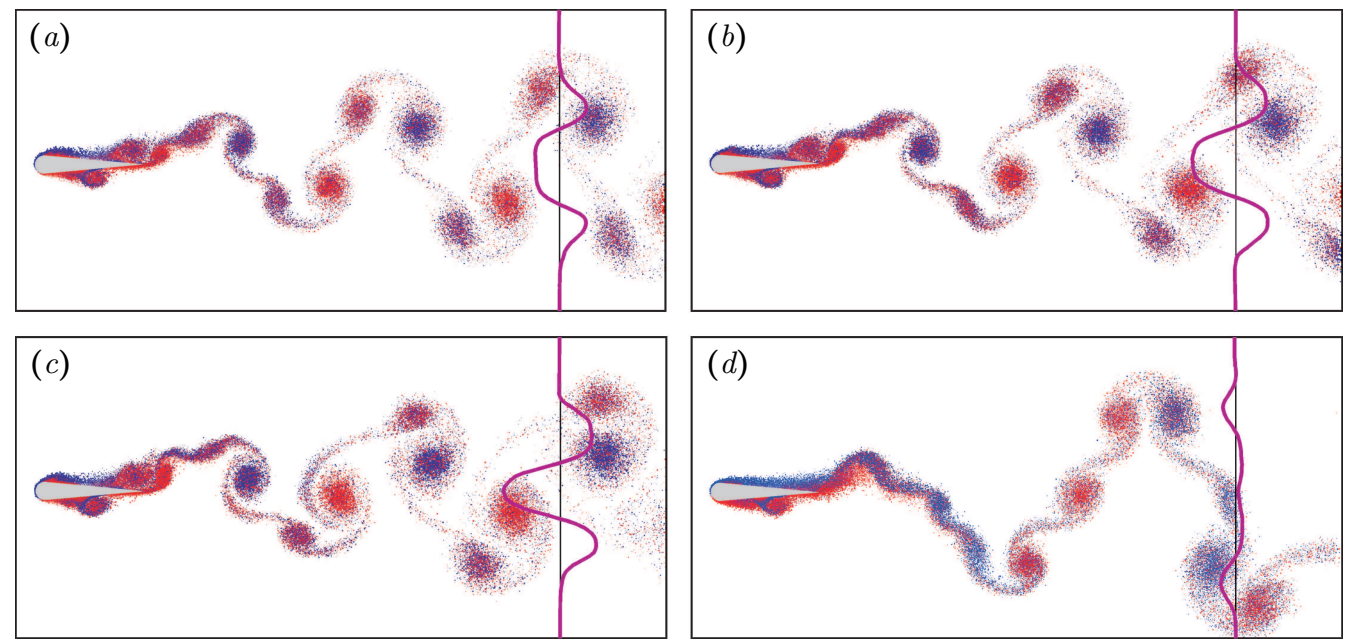

FIGURE 5. Selected wakes of the heaving foil in simulation. Red (blue) corresponds to positive (negative) vorticity, and the magenta lines show average velocity profiles four chord lengths downstream of the trailing edge with the black lines showing the inflow velocity as reference. (a) $2 P$ wake with average drag $\left(\mathrm{St}_{D}=0.10, A_{D}=1.40\right.$, and $\left.\left\langle C_{\mathrm{T}}\right\rangle=-0.03\right),(b) 2 P$ wake immediately below the drag-thrust transition $\left(\mathrm{St}_{D}=0.10, A_{D}=1.60\right.$, and $\left.\left\langle C_{\mathrm{T}}\right\rangle=-0.01\right),(c)$ $2 P$ wake with average thrust $\left(\mathrm{St}_{D}=0.10, A_{D}=1.80\right.$, and $\left.\left\langle C_{\mathrm{T}}\right\rangle=0.02\right)$, see also supplementary video 2 and $(d) 2 P+2 S$ wake $\left(\mathrm{St}_{D}=0.06, A_{D}=1.60\right.$, and $\left.\left\langle C_{\mathrm{T}}\right\rangle=-0.09\right)$.

frequencies and high amplitudes do we find some quantitative differences. The simulations also reproduce two non-overlapping regions with $2 P$ wake measured in the soap film, i.e., one main region and a small island embedded in the von Kármán region. The $2 P$ wakes occur as vortices formed at the leading and trailing edges are shed in turn with a spatial separation sufficiently large to prevent the vortices from merging (Schnipper et al. 2009). From animations of the flow fields it is confirmed that the same process takes place in the simulations. These observations indicate that the boundary layer evolution in the simulation and in the soap film agree well. The agreement in the horizontal width of the $2 P$ island furthermore shows that the spatial threshold for vortex merging is the same in the two methods.

Figure 4 shows the instantaneous thrust and lift for the pitching foil in two representative examples: $(a) \mathrm{St}_{D}=0.12$ and $A_{D}=1.14$ giving rise to a von Kármán wake and average drag $\left\langle C_{\mathrm{T}}\right\rangle=-0.12$ and $(b) \mathrm{St}_{D}=0.22$ and $A_{D}=1.81$ giving rise to an inverted von Kármán wake and average thrust $\left\langle C_{\mathrm{T}}\right\rangle=0.18$. The simulation noise is due to the random walks of the vortex particles (Chorin 1973), and the curves are obtained by applying a 10 points moving average filter to the raw numerical data. The instantaneous lift varies periodically with the period of the foil motion, and the instantaneous drag varies periodically with half of the period as expected from the reflection symmetry in the problem. Average thrust is only produced by the pitching foil when it is oscillated with sufficiently high frequency and amplitude to give rise to an inverted von Kármán wake (figure 3). More precisely the drag-thrust transition (solid blue curve) takes place at $\mathrm{St}_{A}=0.28$ (dashed black curve), which is a higher value than the transition between von Kármán wake and inverted von Kármán wake at $\mathrm{St}_{A}=0.18$ (Schnipper et al. 2009). This observation is in agreement with previous measurements and simulations (GodoyDiana et al. 2008; Bohl \& Koochesfahani 2009; Das et al. 2016), whereas inviscid theory predicts a drag-thrust transition as the wake changes from von Kármán wake to inverted von Kármán (von Kármán \& Burgers 1935). 


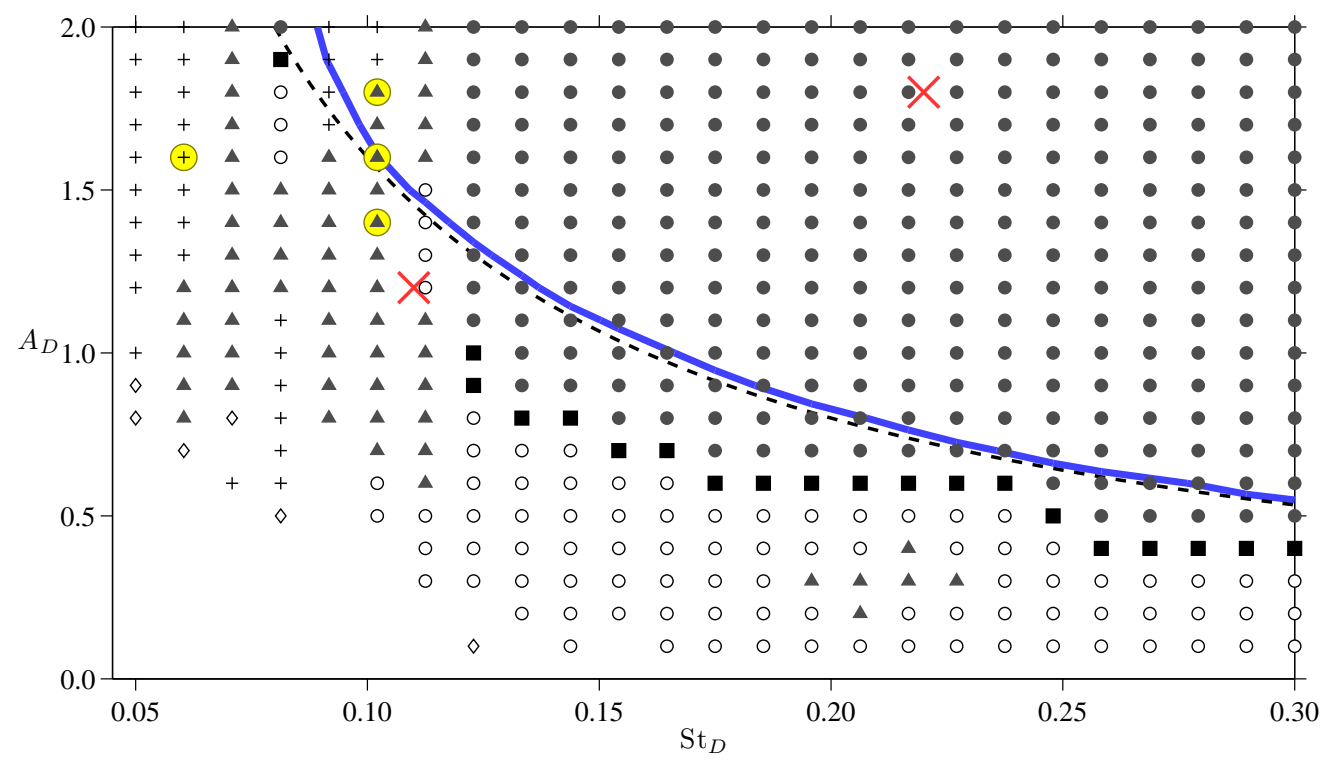

Figure 6. Wake map for the heaving foil in simulation. (+) $2 P+2 S$ wakes, (०) von Kármán wakes, $(\bullet)$ inverted von Kármán wakes, $(\bullet)$ aligned $2 S$ wakes, $(\Delta) 2 P$ wakes and $(\diamond)$ periodic vortex wakes with blurry vorticity regions. The yellow discs indicate the cases in figure 5 and the red crosses the examples shown in figure 7 . The solid blue curve shows the drag-thrust transition, and the dashed black curve shows the contour line with $\mathrm{St}_{A}=0.16$.

\section{Heaving foil in simulation}

The heaving foil forms a variety of $2 P$ wakes (figure 5). For increasing $A_{D}$, the vortex pairs point still more downstream indicating an increasing amount of thrust production. Figure $5(a)$ is in fact a case where the foil experiences average drag, figure $5(b)$ is a case immediately below the drag-thrust transition, and figure $5(c)$ is a case of average thrust production. In addition, figure $5(d)$ shows a $2 P+2 S$ wake where six vortices are shed in each flapping period. A similar wake type is found, for quite similar flapping parameters, behind the pitching foil in the soap film (figure 3 ). However, the two $2 P+2 S$ wakes differ qualitatively in the sense of rotation of the vortex closest to the centre line. The heaving foil sheds three same-signed vortices per flapping half-period. In contrast the pitching foil, cf. figure 3(d) in Schnipper et al. (2009), forms wake arrangements with vortices of alternating sense of rotation.

Similar to the pitching foil, the wake map for the heaving foil consists of a region $\left(\mathrm{St}_{D}>0.1\right)$ dominated by $2 S$ wake types, and a region $\left(\mathrm{St}_{D}<0.1\right)$ that is dominated by more complex wake types (figure 6 ). Further, notice that two non-overlapping regions of $2 P$ wake exist in the wake map: A main region that is located at $0.06<\mathrm{St}_{D}<0.12$ and a small island at $0.19<\mathrm{St}_{D}<0.23$ embedded in the von Kármán wake region. The formation of vortex patches close to the leading and trailing edges appears similar to the pitching foil. From animations of the flow field we measure that a leading edge vortex rolls downstream along the chord with velocity $0.64 U$. Following the simple model by Schnipper et al. (2009) we obtain the theoretical estimates $\mathrm{St}_{D}=0.11$ for the centre of the main $2 P$ region and $\mathrm{St}_{D}=0.22$ for the centre of the $2 P$ island, in reasonably good agreement with the wake map based on the numerical simulations.

For the heaving foil the transition from von Kármán wake to inverted von Kármán wake takes place at lower amplitudes than for the pitching foil (figures 3 and 6). In the 
(a)

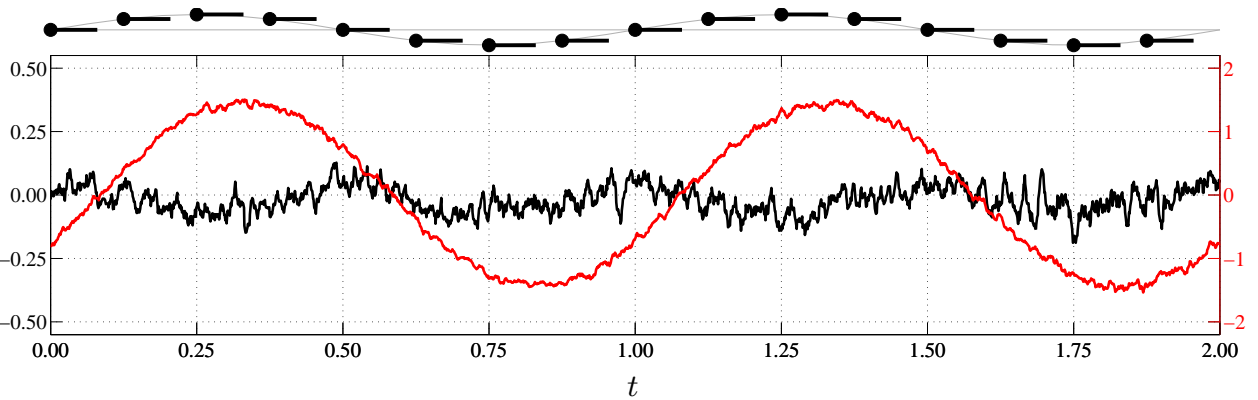

$C_{\mathrm{L}}$

(b)

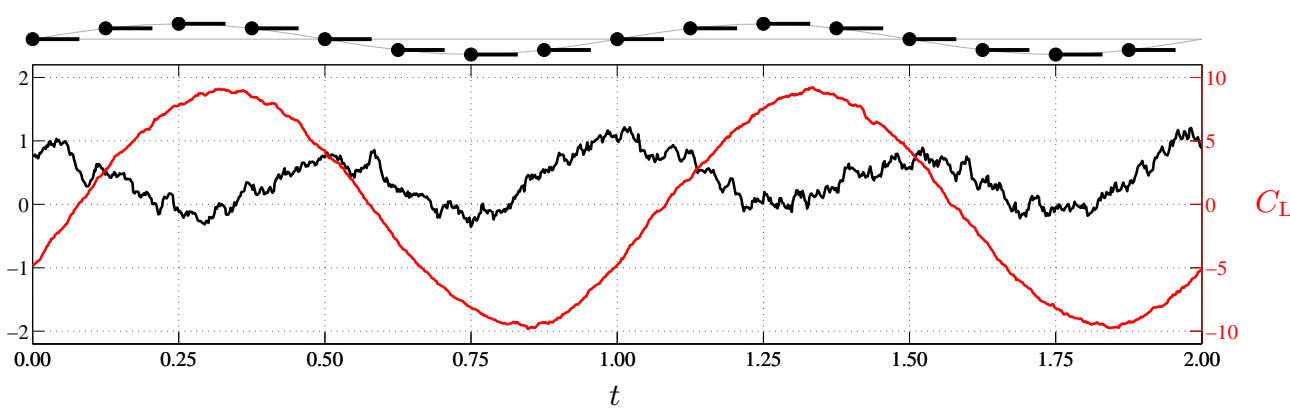

Figure 7. Instantaneous thrust (black) and lift (red) coefficients for the heaving foil in simulation. (a) von Kármán wake with average drag $\left(\mathrm{St}_{D}=0.11\right.$ and $\left.A_{D}=1.20\right)$ and $(b)$ inverted von Kármán wake with average thrust $\left(\mathrm{St}_{D}=0.22\right.$ and $\left.A_{D}=1.80\right)$.

upper-right part of the wake map we find oblique inverted von Kármán wakes (Marais et al. 2012; Das et al. 2016). In the region $\mathrm{St}_{D}<0.12$ the wake map has a complex structure. In addition to $2 P$ wakes we observe exotic $2 P+2 S$ wakes, somewhat scattered in the region. In contrast to the case of the pitching foil, we find a transition from $2 P$ wake to inverted von Kármán wake by increasing $\mathrm{St}_{D}$ for constant amplitude in the range $1.5<A_{D} \leq 2.0$. We do not observe systematically any von Kármán wake to inverted von Kármán wake transition in this region.

Figure 7 shows the instantaneous thrust and lift for the heaving foil in two representative examples: $(a) \mathrm{St}_{D}=0.11$ and $A_{D}=1.20$ giving rise to a von Kármán wake and average drag $\left\langle C_{\mathrm{T}}\right\rangle=-0.03$ and $(b) \mathrm{St}_{D}=0.22$ and $A_{D}=1.80$ giving rise to an inverted von Kármán wake and average thrust $\left\langle C_{\mathrm{T}}\right\rangle=0.39$. We observe both $2 P$ and $2 P+2 S$ in the thrust region of the heaving foil (figure 6 ), and we see that an indication of average thrust production is that the vortex pairs in the $2 P$ wake point in the downstream direction (figure $5 c$ ). The vortex pairs give rise to average jet flow above and below the symmetry line, in contrast to the inverted inverted von Kármán wake with strong average jet flow on the symmetry line (figure $2 d$ ). Similar to the pitching foil we observe that the drag-thrust transition (solid blue curve) closely follows the contour line $\mathrm{St}_{A}=0.16$ (dashed black line). We note that $2 P$ wakes with average thrust, interpreted from slices through three-dimensional vortex rings, have been measured behind freely swimming fish such as bluegill sunfish (Drucker \& Lauder 2002) and eel (Tytell \& Lauder 2004). It will be interesting to explore to what extent the results of the present study are influenced by three-dimensional effects. 


\section{Conclusions}

The agreement between the vortex particle method and the measurements in the soap film tunnel shows that the soap film technique is a versatile tool to visualise flow structures and to relate them to the corresponding two-dimensional, incompressible Newtonian flows. Only at high oscillation frequency and amplitude did we observe differences, presumably due to the compressibility of the soap film. The wake maps summarising the wake types for pitching and heaving turned out to be qualitatively similar. The main difference in the wake structures and thrust production between the two different kinematic modes turned out to be relation between the drag-thrust transition and the changes in wake structure at low oscillation frequency and high amplitude, where the heaving foil displayed drag-thrust transition in a $2 P$ wake region with jets symmetrically above and below the symmetry line.

We would like to dedicate this paper to the memory of our dear friend and colleague Professor Hassan Aref. Also we are thankful to Jane Wang for fruitful discussions and to Erik Hansen for his careful work in constructing the experimental set-up.

\section{REFERENCES}

Bohl, D. G. \& Koochesfahani, M. M. 2009 MTV measurements of the vortical field in the wake of an airfoil oscillating at high reduced frequency. J. Fluid Mech. 620, 63-88.

Buchholz, J. H. J. \& Smits, A. J. 2008 The wake structure and thrust performance of a rigid low-aspect-rato pitching panel. J. Fluid Mech. 603, 331-365.

Carrier, J., Greengard, L. \& Rokhlin, V. 1988 A fast adaptive multipole algorithm for particle simulations. SIAM J. Sci. Stat. Comput. 9 (4), 669-686.

Chomaz, J.-M. \& Cathalau, B. 1990 Soap films as two-dimensional classical fluids. Phys. Rev. A 41, 2243-2245.

Chorin, A. J. 1973 Numerical study of slightly viscous flow. J. Fluid Mech. 57 (4), 785-796.

Couder, Y. \& BASDEvant, C. 1986 Experimental and numerical study of vortex couples in two-dimensional flows. J. Fluid Mech. 173, 225-251.

Couder, Y., Chomaz, J. M. \& Rabaud, M. 1989 On the hydrodynamics of soap films. Physica $D$ 37, 384-405.

Das, A., Shukla, R. K. \& Govardhan, R. N. 2016 Existence of a sharp transition in the peak propulsive efficiency of a low-Re pitching foil. J. Fluid Mech. 800, 307-326.

Drucker, E. G. \& Lauder, G. V. 2002 Experimental hydrodynamics of fish locomotion: Functional insights from wake visualization. Int. Comp. Biol. 42, 243-257.

Gharib, M. \& Derango, P. 1989 A liquid film (soap film) tunnel to study two-dimensional laminar and turbulent shear flows. Physica D 37, 406-416.

Godoy-Diana, R., Aider, J.-L. \& Wesfreid, J. E. 2008 Transitions in the wake of a flapping foil. Phys. Rev. E 77, 016308.

Hejlesen, M. M., Koumoutsakos, P., Leonard, A. \& Walther, J. H. 2015 Iterative Brinkman penalization for remeshed vortex methods. J. Comput. Phys. 280, 547-562.

Jaworski, J. W. \& GordnieR, R. 2015 Thrust augmentation of flapping airfoils in low Reynolds number flow using a flexible membrane. J. Fluids and Structures 52, 199-209.

von KÁrmán, T. \& Burgers, J. M. 1935 General aerodynamic theory - perfect fluids. In Aerodynamic Theory II (ed. W. F. Durand). Dover.

Koochesfahani, M. M. 1986 Wake of an oscillating airfoil. Phys. Fluids 29, 2776.

Koochesfahani, M. M. 1989 Vortical patterns in the wake of an oscillating airfoil. AIAA J. 27, 1200-1205.

Larsen, A. \& Walther, J. H. 1997 Aeroelastic analysis of bridge girder sections based on discrete vortex simulations. J. Wind Eng. Ind. Aerodyn. 67-68, 253-265.

Lighthill, M. J. 1969 Hydromechanics of aquatic animal propulsion. Annu. Rev. Fluid Mech. 1, 413-446. 
Wake structure and thrust generation of a flapping foil in two-dimensional flow 11

Mackowski, A. W. \& Williamson, C. H. K. 2015 Direct measurement of thrust and efficiency of an airfoil undergoing pure pitching. J. Fluid Mech. 765, 524-543.

Marais, C., Thiria, B., Wesfreid, J. E. \& Godoy-Diana, R. 2012 Stabilizing effect of flexibility in the wake of a flapping foil. J. Fluid Mech. 710, 659-669.

Rasmussen, J. T., Hejlesen, M. M., Larsen, A. \& Walther, J. H. 2010 Discrete vortex method simulations of the aerodynamic admittance in bridge aerodynamics. J. Wind Eng. Ind. Aerodyn. 98, 754-766.

Rivera, M., Vorobieff, P. \& Ecke, R. E. 1998 Turbulence in flowing soap films: Velocity, vorticity, and thickness fields. Phys. Rev. Lett. 81 (7), 1417-1420.

Rutgers, M. A., Wu, X. L. \& Daniel, W. B. 2001 Conducting fluid dynamics experiments with vertically falling soap films. Rev. Sci. Instr. 72, 3025-3037.

Schnipper, T., Andersen, A. \& Bohr, T. 2009 Vortex wakes of a flapping foil. J. Fluid Mech. 644, 411-423.

Schnipper, T., Tophøj, L., Andersen, A. \& Bohr, T. 2010 Japanese fan flow. Phys. Fluids 22, 091102.

Sfakiotakis, M., Lane, D. M. \& Davies, J. B. C. 1999 Review of fish swimming modes for aquatic locomotion. IEEE J. Oceanic Eng. 24, 237-252.

Triantafyllou, M. S., Triantafyllou, G. S. \& Gopalkrishnan, R. 1991 Wake mechanics for thrust generation in oscillating foils. Phys. Fluids A 3, 2835-2837.

Triantafyllou, M. S., Triantafyllou, G. S. \& Yue, D. K. P. 2000 Hydrodynamics of fishlike swimming. Annu. Rev. Fluid Mech. 32, 33-53.

Tytell, E. D. \& Lauder, G. V. 2004 The hydrodynamics of eel swimming I. Wake structure. J. Exp. Biol. 207 (11), 1825-1841.

Walther, J. H. \& LARSEn, A. 1997 Discrete vortex method for application to bluff body aerodynamics. J. Wind Eng. Ind. Aerodyn. 67-68, 183-193.

Williamson, C. H. K. \& Roshko, A. 1988 Vortex formation in the wake of an oscillating cylinder. J. Fluids and Structures 2, 335-381.

Wu, J. C. 1981 Theory for aerodynamic force and moment in viscous flows. AIAA J. 19, 432-441.

Zhang, J., Childress, S., Libchaber, A. \& Shelley, M. 2000 Flexible filaments in a flowing soap film as a model for one-dimensional flags in a two-dimensional wind. Nature 408, 835-839. 